Proceedings

\title{
Future Climate Alters Pathogens-Microbiome Co-occurrence Networks in Wheat Straw Residues during Decomposition ${ }^{+}$
}

\author{
Sara Fareed Mohamed Wahdan 1,2, François Buscot ${ }^{1,3}$ and Witoon Purahong 1,* \\ 1 Department of Soil Ecology, UFZ-Helmholtz Centre for Environmental Research, Theodor-Lieser-Str. 4, \\ 06120 Halle (Saale), Germany; sara-fareed-mohamed.wahdan@ufz.de (S.F.M.W.); \\ francois.buscot@ufz.de (F.B.) \\ 2 Department of Botany, Faculty of Science, Suez Canal University, Ismailia 41522, Egypt \\ 3 German Centre for Integrative Biodiversity Research (iDiv) Halle-Jena-Leipzig, Deutscher Platz 5e, \\ 04103 Leipzig, Germany \\ * Correspondence: witoon.purahong@ufz.de; Tel.: +49-345-558-5207 \\ + Presented at the 1st International Electronic Conference on Microbiology, 2-30 November 2020; Available \\ online: https://ecm2020.sciforum.net/.
}

Published: 11 January 2021

\begin{abstract}
The return of plant residues to the ground is used to promote soil carbon sequestration, improve soil structure, reduce evaporation, and help to fix additional carbon dioxide in the soil. The microbial communities with diverse ecological functions that colonize plant residues during decomposition are expected to be highly dynamic. We aimed to characterize microbial communities colonizing wheat straw residues and their ecological functions during the early phase of straw decomposition. The experiment, run in Central Germany, was conducted in a conventional farming system under both ambient conditions and a future climate scenario expected in 50-70 years from now. We used MiSeq illumina sequencing and network analysis of bacterial 16S rRNA and fungal ITS genes. Our results show that future climate alters the dynamics of bacterial and fungal communities during decomposition. We detected various microbial ecological functions within wheat straw residues such as plant growth-promoting bacteria, $\mathrm{N}$-fixing bacteria, saprotrophs, and plant pathogenic fungi. Interestingly, plant pathogenic fungi dominated $(\sim 87 \%$ of the total sequences) within the wheat residue mycobiome under both ambient and future climate conditions. Therefore, we applied co-occurrence network analysis to predict the potential impacts of climate change on the interaction between pathogenic community and other bacterial and fungal microbiomes. The network under ambient climate consisted of 91 nodes and 129 correlations (edges). The highest numbers of connections were detected for the pathogens Mycosphaerella tassiana and Neosetophoma rosigena. The network under future climate consisted of 100 nodes and 170 correlations. The highest numbers of connections were detected for the pathogens Pseudopithomyces rosae and Gibellulopsis piscis. We conclude that the future climate significantly changes the interactions between plant pathogenic fungi and other microorganisms during the early phrase of decomposition.
\end{abstract}

Keywords: future climate; litter decomposition; Illumina Miseq; pathogens; microbial community composition

\section{Introduction}

Soil microbial communities are the engines of plant residues decomposition. They convert litter detritus into $\mathrm{CO}_{2}$ and release nutrients for plant growth [1]. Litter residues are a transient substrate 
between plant that decompose over one or more cropping season and soil [2]. Therefore, we expect strong microbial dynamics and diverse ecological functions in such residues over time. Plant residues can be considered as one of the most important sources of plant pathogens [2]. These residue-borne pathogens can cause a significant loss of crop yield and contamination by mycotoxin [3]. These residue-borne pathogens are known to interact with other microorganisms living in the same habitat and such interactions can determine the success of the residue-borne pathogens to cause disease to crop plants at the next growing season [4]. Thus, understanding the interactions between residueborne pathogens and other microorganisms living in the same habitat can be useful for developing the effective biological control of residue-borne diseases.

Climate change, in terms of increasing temperatures and decreasing precipitation patterns, will have an unpredicted impact on soil microbial diversity and community compositions. However, the effects of climate change on the diversity and dynamics of wheat residue-inhabiting microbiomes are not well understood [5]. The effects of climate change on the interactions between residue-borne pathogens and other microorganisms sharing the same habitat are still unknown.

Here, we aimed to determine the dynamics of microbial communities (bacteria and fungi) colonizing wheat straw under ambient and future climate conditions. We also determined the potential metabolic and ecological guilds of the colonizing microbes. Lastly, we investigated the cooccurrence network patterns between plant pathogenic fungi and other microbes inhabiting wheat residues at the early stage of decomposition. Our goal was to explore whether climate change might impair the balance of the plant residue microbiome toward enhanced pathogenicity.

\section{Methods}

\subsection{Study Site, Experimental Setup, and Design}

The study was conducted within the Global Change Experimental Facility (GCEF) at the field research station of the Helmholtz Centre for Environmental Research in Bad Lauchstädt, SaxonyAnhalt, Germany $\left(51^{\circ} 22^{\prime} 60 \mathrm{~N}, 11^{\circ} 50^{\prime} 60 \mathrm{E}, 118 \mathrm{~m}\right.$ a.s.l.). The GCEF is a large field experiment for the investigation of the consequences of climate change on ecosystem processes under different land use types [6]. Half of the field plots are subjected to a future climate scenario based on several models of climate change predicting the climate in Central Germany for the years between 2070 and 2100. The future climate scenario is characterized by a reduction in precipitation by $\sim 20 \%$ in the summer months and increasing by $\sim 10 \%$ in spring and autumn, as well as an increase in the mean daily temperature by $\sim 0.55{ }^{\circ} \mathrm{C}$. The wheat straw decomposition experiment was performed on the conventional farming plots under both ambient (five plots) and future climate (five plots) conditions.

\subsection{Litterbag Preparation, Field Incorporation, and Sampling}

The straw left over (10 $\mathrm{cm}$ aboveground) from harvested winter wheat (Triticum aestivum L.) was sampled from each GCEF field plot, oven-dried at $25^{\circ} \mathrm{C}$ for 3 days to normalize the moisture content, and then aliquots of $10 \mathrm{~g}$ were enclosed in litter bags $(20 \times 15 \mathrm{~cm}, 5 \mathrm{~mm}$ mesh size). Three litter bags were returned back to each field plot (five ambient conventional farming plots and five future conventional farming plots). The first sampling of litterbags occurred at the onset of the experiment (0 days). Two further samples were collected, the first 30 days, and the second 60 days after field incorporation.

\subsection{DNA Extraction, PCR, and Illumina Miseq Sequencing}

At harvest, wheat straw from each bag was homogenized and used for DNA extraction with a DNeasy PowerSoil kit (Qiagen, Valencia, CA, USA) run according to the manufacturer's instructions. For identification of bacterial taxa, the V5-V7 region of the bacterial 16S rRNA was amplified using the forward primer BAC799F (5'-AACMGGATTAGATACCCKG-3') [7] and the reverse primer BAC1193r (5'-ACGTCATCCCCACCTTCC-3') [8]. For identification of the fungal taxa, the internal transcribed spacer 2 (ITS2) region of the rRNA was amplified using the fungal-specific forward primer fITS7 (5'-GTGARTCATCGAATCTTTG-3') [9] and the reverse primer ITS4 (5'- 
TCCTCCGCTTATTGATATGC-3') [10]. The amplicons were then sequenced using MiSeq $2 \times 300 \mathrm{bp}$ paired-end strategy.

\subsection{Sequence Processing and Statistics}

The sequences corresponding to the forward and reverse primers were trimmed from the demultiplexed raw reads using cutadapt [11]. Merging of the pair-end raw reads was done using the simple Bayesian algorithm with a threshold of 0.6 and a minimum overlap of 20 nucleotides, as implemented in PANDAseq [12]. Potential chimeras were removed using UCHIME [13] as implemented in MOTHUR [14]. High-quality reads were clustered into operational taxonomic units (OTUs) using cd-hit-est 4.6.2 [15] at a threshold of 97\% pairwise similarity. Bacterial 16S rRNA OTU representative sequences were assigned against the SILVA v132 reference sequence database [16]. Fungal ITS representative sequences were assigned against UNITE v7 sequence database [17]. Ecological and metabolic functions were predicted for detected bacterial OTUs using FAPROTAX [18], the functional Annotation tool of Prokaryotic Taxa v.1.1 and with FUNGuild [19] for fungi. Permutational multivariate analysis of variance (NPMANOVA) [20] based on Jaccard distance was performed to test dynamics of bacterial and fungal communities at each sampling time $(0,30$, and 60 days) subjected to ambient and future climate treatments. Interaction between plant pathogenic fungi and other microorganisms inhabiting wheat residues at the early stage of decomposition were analyzed using non-parametric Spearman's rank correlation $(p<0.05)$. Networks of OTUs and their adjacent nodes were visualized with Cytoscape V. 3.8.0 [21].

\section{Results}

\subsection{Climate Change Significantly Alters Microbial Community Composition during Early Stages of Wheat} Straw Decomposition

Microbial communities displayed different dynamic patterns under the ambient and future climate treatments during the early stages of the wheat residue decomposition. Under the ambient climate treatment, the NMDS analysis showed that communities overlapped at the three sampling times for fungi and at 30 and 60 days for bacteria. In contrast, under the future climate treatment, the microbial communities at each sampling time were highly distinct from each other (Figure 1C,D).

Ecological functions were predicted for detected OTUs using the FAPROTAX and FUNGuild databases for bacteria and fungi, respectively. We found that plant pathogenic fungi dominated the fungal communities (relative abundance $79.8-92.7 \%$ ) at all time points and under both climate treatments. Therefore, we aimed to investigate the co-occurrence pathogen between plant pathogenic fungi and other microbial communities. 

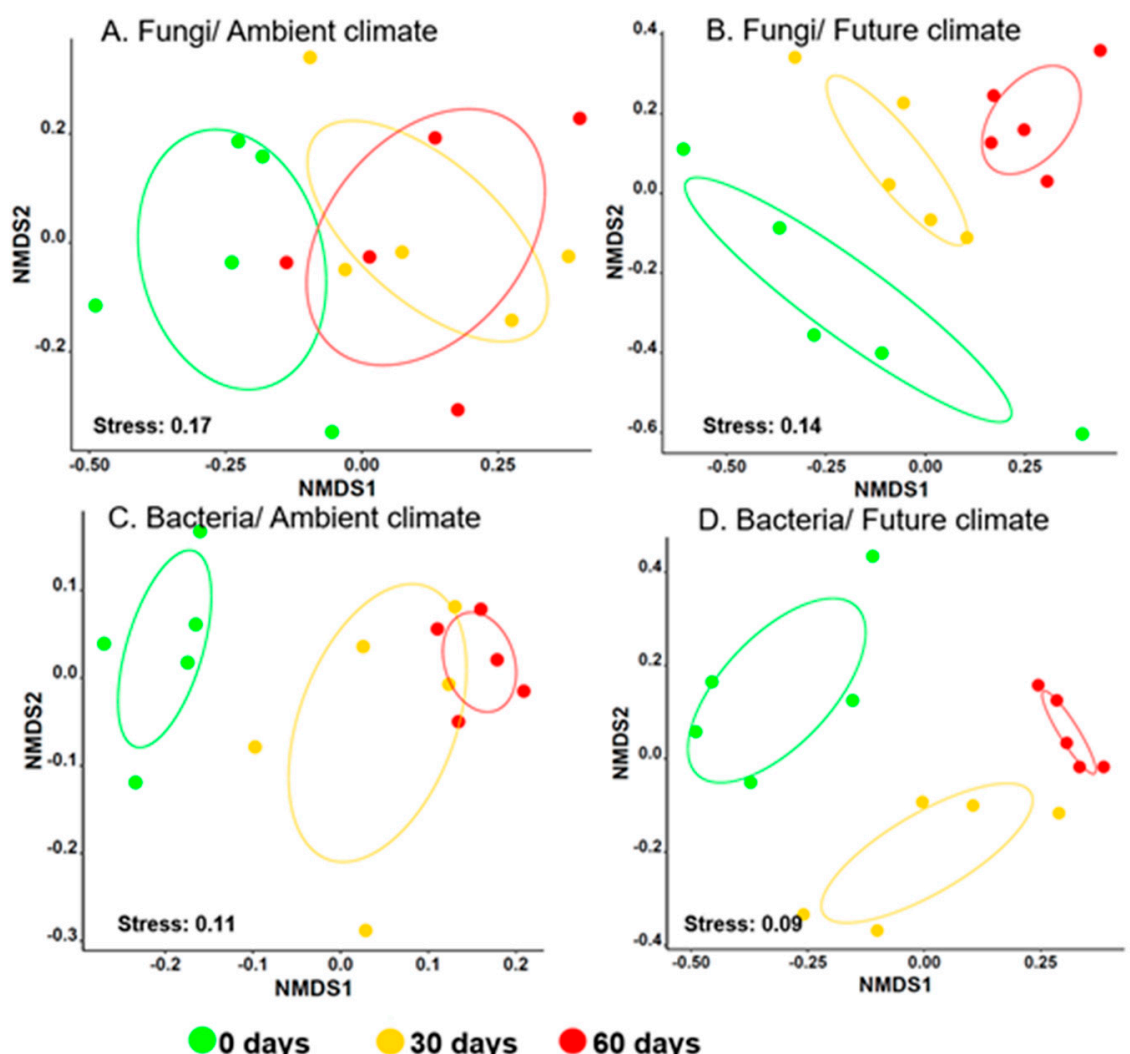

0 days 30 days

60 days

Figure 1. Non-metric multidimensional scaling (NMDS) ordination diagrams of the fungal community colonizing wheat straw residues in (A) ambient and (B) future climate treatments; the bacterial community in (C) ambient and (D) future climate treatments.

\subsection{Co-Occurrence Patterns between Microbes Colonizing Wheat Straw Residues}

We explored co-occurrence network patterns of microbial communities inhabiting wheat straw under ambient and future climate (Figure 2) using network analysis based on strong and significant correlations (using non-parametric Spearman's rank). Each network comprised positive and negative co-occurrences. The ambient climate network had a total of 91 nodes and 129 edges, where 51 had negative correlations, while the future climate network consisted of 100 nodes and 170 edges, where 37 had negative correlations. Under both ambient and future climate regimes, some pathogenic fungi had the highest number of connections (potential keystone taxa) with bacterial and fungal communities, for instance, Mycosphaerella tassiana and Plectosphaerella oratosquillae under the ambient climate and Pseudopithomyces rosae and Gibellulopsis piscis under the future climate. Apart from the changes in key stone plant pathogen fungi, our results also indicate the changes in the interacting microbial taxa and type of interactions (positive to negative, positive to no interaction, negative to positive and negative to no interaction) due to different climate treatments. 


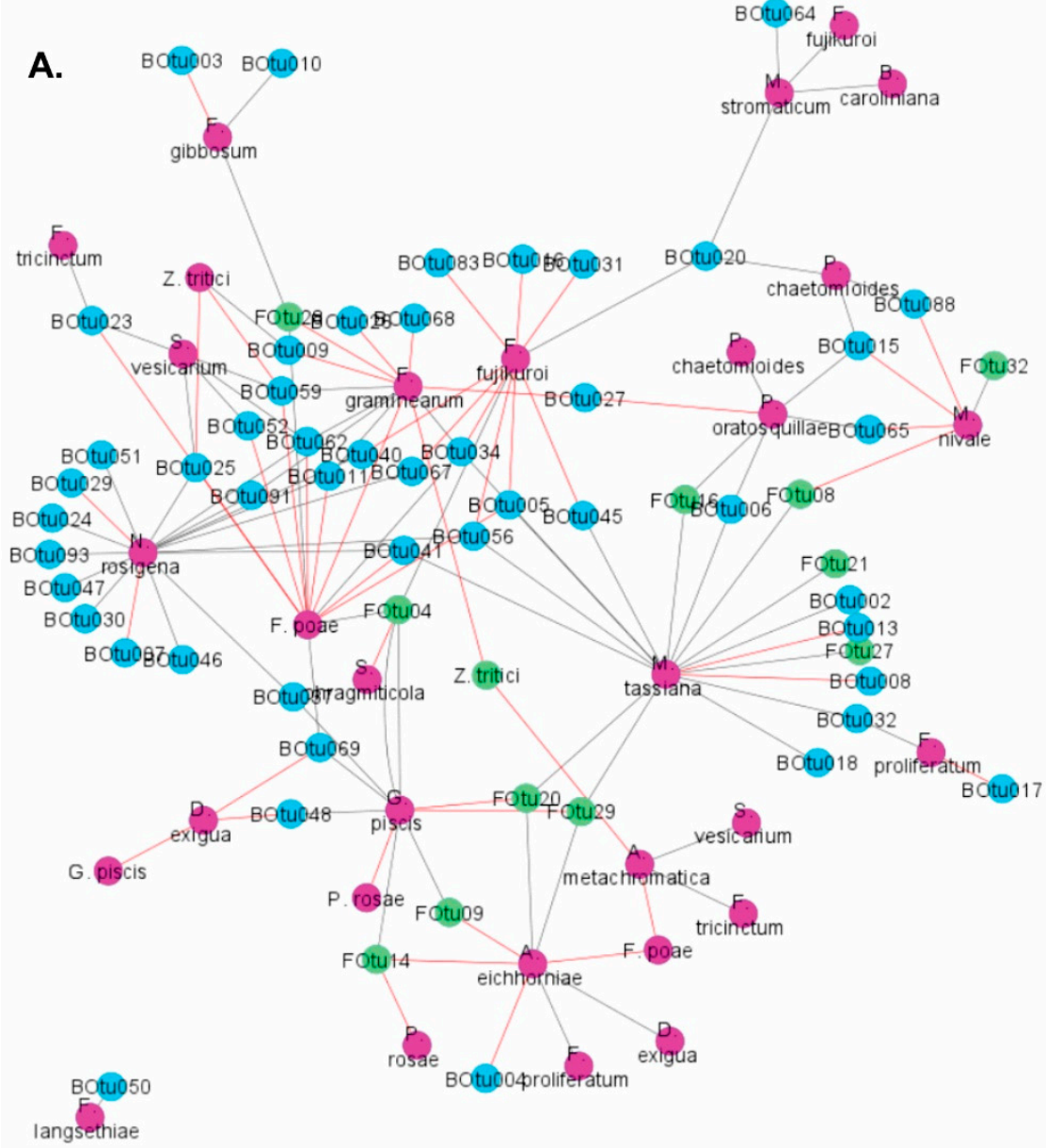




\section{Conclusions}

Our analyses showed a potentially high impact of climate change on microbial community dynamics in wheat residue at the early stage of decomposition. We found fungal pathogens to be dominant over other guilds under both ambient and future climates. Those plant pathogenic fungi co-occur and potentially interact with other microbes colonizing wheat residues. Climate treatments significantly change the key stone plant pathogenic fungi as well as the interacting microbial taxa and type of interactions. This indicates that interactions between plant pathogenic fungi and other microorganisms where shaped by climate treatment. Therefore, current effective disease control strategies may not stay effective in the future. The interaction between plant pathogenic fungi and other microorganisms in the future climate system should be considered to develop the effective disease control of residue-borne diseases.

Acknowledgments: We thank the Helmholtz Association, the Federal Ministry of Education and Research, the State Ministry of Science and Economy of Saxony-Anhalt, and the State Ministry for Higher Education, Research and the Arts Saxony for funding the Global Change Experimental Facility (GCEF) project. We also thank the staff of the Bad Lauchstädt Experimental Research Station (especially Ines Merbach and Konrad Kirsch) for their work in maintaining the plots and infrastructures of the GCEF, and Martin Schädler for coordinating the project, Stefan Klotz, Harald Auge, and Thomas Reitz for their roles in setting up the GCEF. The sequencing data were analyzed at the High-Performance Computing (HPC) Cluster EVE, a joint effort between the Helmholtz Centre for Environmental Research-UFZ and the German Centre for Integrative Biodiversity Research (iDiv) Halle-JenaLeipzig.

\section{References}

1. Krishna, M.P.; Mohan, M. Litter decomposition in forest ecosystems: A review. Energy Ecol. Environ. 2017, 2, 236-249, doi:10.1007/s40974-017-0064-9.

2. Kerdraon, L.; Laval, V.; Suffert, F. Microbiomes and pathogen survival in crop residues, an ecotone between plant and soil. Phytobiomes J. 2019, 3, 246-255, doi:10.1094/pbiomes-02-19-0010-rvw.

3. Duba, A.; Goriewa-Duba, K.; Wachowska, U. A Review of the Interactions between Wheat and Wheat Pathogens: Zymoseptoria tritici, Fusarium spp. and Parastagonospora nodorum. Int. J. Mol. Sci. 2018, 19, 1138, doi:10.3390/ijms19041138.

4. Kerdraon, L.; Barret, M.; Laval, V.; Suffert, F. Differential dynamics of microbial community networks help identify microorganisms interacting with residue-borne pathogens: The case of Zymoseptoria tritici in wheat. Microbiome 2019, 7, 125, doi:10.1186/s40168-019-0736-0.

5. Fareed Mohamed Wahdan, S.; Hossen, S.; Tanunchai, B.; Schädler, M.; Buscot, F.; Purahong, W. Future climate significantly alters fungal plant pathogen dynamics during the early phase of wheat litter decomposition. Microorganisms 2020, 8, 908, doi:10.3390/microorganisms8060908.

6. Schädler, M.; Buscot, F.; Klotz, S.; Reitz, T.; Durka, W.; Bumberger, J.; Merbach, I.; Michalski, S.G.; Kirsch, K.; Remmler, P.; et al. Investigating the consequences of climate change under different land-use regimes: A novel experimental infrastructure. Ecosphere 2019, 10, e02635.

7. Chelius, M.K.; Triplett, E.W. The diversity of archaea and bacteria in association with the roots of Zea mays L. Microb. Ecol. 2001, 41, 252-263, doi:10.1007/s002480000087.

8. Bodenhausen, N.; Horton, M.W.; Bergelson, J. Bacterial communities associated with the leaves and the roots of Arabidopsis thaliana. PLoS ONE 2013, 8, e56329, doi:10.1371/journal.pone.0056329.

9. Ihrmark, K.; Bodeker, I.T.; Cruz-Martinez, K.; Friberg, H.; Kubartova, A.; Schenck, J.; Strid, Y.; Stenlid, J.; Brandstrom-Durling, M.; Clemmensen, K.E.; et al. New primers to amplify the fungal ITS2 regionevaluation by 454-sequencing of artificial and natural communities. FEMS Microbiol. Ecol. 2012, 82, 666677, doi:10.1111/j.1574-6941.2012.01437.x.

10. White, T.J.; Bruns, T.D.; Lee, S.B.; Taylor, J.W. Amplification and direct sequencing of fungal ribosomal RNA genes for phylogenetics. In PCR Protocols: A Guide to Methods and Applications; Innis, M.A., Gelfand, D.H., Sninsky, J.J., White, T.J., Eds.; Academic Press: Cambridge, MA, USA, 1990; pp. pp. 315-322.

11. Martin, M. Cutadapt removes adapter sequences from high-throughput sequencing reads. EMBnet J. 2011, 17, 10-12, doi:10.14806/ej.17.1.200.

12. Masella, A.P.; Bartram, A.K.; Truszkowski, J.M.; Brown, D.G.; Neufeld, J.D. PANDAseq: Paired-end assembler for illumina sequences. BMC Bioinform. 2012, 13, doi:10.1186/1471-2105-13-31. 
13. Edgar, R.C.; Haas, B.J.; Clemente, J.C.; Quince, C.; Knight, R. UCHIME improves sensitivity and speed of chimera detection. Bioinformatics 2011, 27, 2194-2200, doi:10.1093/bioinformatics/btr381.

14. Schloss, P.D.; Westcott, S.L.; Ryabin, T.; Hall, J.R.; Hartmann, M.; Hollister, E.B.; Lesniewski, R.A.; Oakley, B.B.; Parks, D.H.; Robinson, C.J.; et al. Introducing mothur: Open-source, platform-independent, community-supported software for describing and comparing microbial communities. Appl Environ. Microbiol. 2009, 75, 7537-7541, doi:10.1128/AEM.01541-09.

15. Fu, L.; Niu, B.; Zhu, Z.; Wu, S.; Li, W. CD-HIT: Accelerated for clustering the next-generation sequencing data. Bioinformatics 2012, 28, 3150-3152, doi:10.1093/bioinformatics/bts565.

16. Quast, C.; Pruesse, E.; Yilmaz, P.; Gerken, J.; Schweer, T.; Yarza, P.; Peplies, J.; Glockner, F.O. The SILVA ribosomal RNA gene database project: Improved data processing and web-based tools. Nucleic Acids Res. 2013, 41, D590-D596, doi:10.1093/nar/gks1219.

17. Kõljalg, U.; Nilsson, R.H.; Abarenkov, K.; Tedersoo, L.; Taylor, A.F.S.; Bahram, M.; Bates, S.T.; Bruns, T.D.; Bengtsson-Palme, J.; Callaghan, T.M.; et al. Towards a unified paradigm for sequence-based identification of fungi. Mol. Ecol 2013, 22, 5271-5277, doi: 10.1111/mec.12481.

18. Louca, S.; Parfrey, L.W.; Doebeli, M. Decoupling function and taxonomy in the global ocean microbiome. Science 2016, 353, 1272-1277, doi:10.1126/science.aaf4507.

19. Nguyen, N.H.; Song, Z.; Bates, S.T.; Branco, S.; Tedersoo, L.; Menke, J.; Schilling, J.S.; Kennedy, P.G. FUNGuild: An open annotation tool for parsing fungal community datasets by ecological guild. Fungal Ecol. 2016, 20, 241-248, doi:10.1016/j.funeco.2015.06.006.

20. Anderson, M.J. A new method for non-parametric multivariate analysis of variance. Austral Ecol 2001, 26, 32-46.

21. Shannon, P.; Markiel, A.; Ozier, O.; Baliga, N.S.; Wang, J.T.; Ramage, D.; Amin, N.; Schwikowski, B.; Ideker, T. Cytoscape: A software environment for integrated models of biomolecular interaction networks. Genome Res. 2003, 13, 2498-2504, doi:10.1101/gr.1239303.

Publisher's Note: MDPI stays neutral with regard to jurisdictional claims in published maps and institutional affiliations.

(C) 2021 by the authors. Licensee MDPI, Basel, Switzerland. This article is an open access article distributed under the terms and conditions of the Creative Commons Attribution (CC BY) license (http://creativecommons.org/licenses/by/4.0/). 